OPEN ACCESS

Edited by:

Amit K. Tiwari,

University of Toledo, USA

Reviewed by:

Alessandro Isidori

Aormn Hospital, Italy

Dev Prasad,

Fresenius Kabi, USA

Jozsef Dudas,

Innsbruck Medical University, Austria

*Correspondence: Jingxin Mo

mojingx3@mail.sysu.edu.cn Honglian Dai

daihonglian@whut.edu.cn

tThese authors have contributed equally in this paper.

Specialty section:

This article was submitted to Pharmacology of Anti-Cancer Drugs,

a section of the journal

Frontiers in Pharmacology

Received: 29 October 2016

Accepted: 24 January 2017

Published: 14 February 2017

Citation:

Li B, Li Q, Mo J and Dai H (2017)

Drug-Loaded Polymeric

Nanoparticles for Cancer Stem Cell

Targeting. Front. Pharmacol. 8:51.

doi: 10.3389/fphar.2017.00051

\section{Drug-Loaded Polymeric Nanoparticles for Cancer Stem Cell Targeting}

\author{
Binbin $\mathrm{Li}^{1,2 \dagger}$, Qinghua $\mathrm{Li}^{3 \dagger}$, Jingxin $\mathrm{Mo}^{4,5 *}$ and Honglian Dai ${ }^{1,2 *}$ \\ 1 State Key Laboratory of Advanced Technology for Materials Synthesis and Processing, Wuhan University of Technology, \\ Wuhan, China, ${ }^{2}$ Biomedical Materials and Engineering Research Center of Hubei Province, Wuhan, China, ${ }^{3}$ Department of \\ Neurology, Affiliated Hospital of Guilin Medical University, Guilin, China, ${ }^{4}$ Key Laboratory for Stem Cells and Tissue \\ Engineering (Sun Yat-sen University), Ministry of Education, Guangzhou, China, ${ }^{5}$ Department of Histology and Embryology, \\ Zhongshan School of Medicine, Sun Yat-sen University, Guangzhou, China
}

Cancer stem cells (CSCs) have been reported to play critical roles in tumor initiation, propagation, and regeneration of cancer. Nano-size vehicles are employed to deliver drugs to target the CSCs for cancer therapy. Polymeric nanoparticles have been considered as the most efficient vehicles for drug delivery due to their excellent pharmacokinetic properties. The CSCs specific antibodies or ligands can be conjugated onto the surface or interior of nanoparticles to successfully target and finally eliminate CSCs. In this review, we focus on the approaches of polymeric nanoparticles design for loading drug, and their potential application for CSCs targeting in cancer therapy.

Keywords: cancer stem cell, drug-loaded, polymeric nanoparticle, cancer therapy

\section{INTRODUCTION}

Cancer is widely viewed as the major reason of death in the world (El-Serag, 2001). Although decades of efforts of research and billions of funding have enhanced our understanding of the underlying mechanisms of tumorigenesis, cancer caused mortality remains running at a high level nowadays (Oishi and Wang, 2011). Recent studies have suggested that, the growth of tumors is similar to that of normal proliferative tissues, fueled by limited numbers of dedicated stem cells that are capable of self-renewal, called cancer stem cells (CSCs) (Reya et al., 2001; Clarke et al., 2006; Adams and Strasser, 2008; Dick, 2009; Wilson et al., 2009; He et al., 2016).

The concept of CSCs was introduced firstly in Bonnet and Dick (1997). CSCs have been identified in multiple malignancies, which are capable of self-renewal, and drive the propagation and development of a tumor (Jordan et al., 2006; Rosen and Jordan, 2009; O’Brien et al., 2010; Clevers, 2011). Due to these crucial roles, failure to efficiently eliminate CSCs during conventional therapy may lead to tumor relapse and metastases. Thus, CSCs are considered as a critical issue to be addressed in the cancer therapy (Dean et al., 2005; Li et al., 2015). Quite a lot of efforts have been put into the study of anti-CSCs strategies to completely eliminate the CSCs population, and some approaches have gained encouraging therapeutic outcomes, such as inhibiting the signaling pathways related to self-renewal to interfere the CSCs proliferation and tumor growth, breaking the tumor microenvironment to decrease the interaction between CSCs and cytokines, and targeting the markers on CSCs surface to locate and destroy CSCs etc. (Beck and Blanpain, 2013; Chen et al., 2013; Lu et al., 2016). Within these strategies, the hottest research in the field of 
cancer therapy is CSCs targeting, because of its ability to improve the survival of cancer patients, especially patients with drug resistance in recent years (Zhou et al., 2009).

However, the sensitivity of the drug changed usually in the tumor due to either genetic or epigenetic alterations (Zahreddine and Borden, 2013). This would lead to acquired resistance following the change of gene expression patterns, and eventually drug selection during cancer treatment. Drug resistance results in a series of problems including: the increase of CSCs population, disease relapse and tumor metastasis during the period of cancer treatment (Zhou et al., 2009; Takebe et al., 2011).

A large number of efforts have been done to minimize the harmful impacts of drugs during the process of cancer therapy (Vinogradov and Wei, 2012): (a) preventing the side effects on the nearby cells and tissues; (b) increasing the drug accumulation and efficacy in the lesion; (c) developing novel drug delivery and targeting systems.

The key factor to solve these issues above is to allow the CSCs exposed to the high enough drug concentration in cellular environment. Numerous novel drug delivery strategies have been developed with the rapid advances of nanotechnology. The nanosized drug delivery system can be designed to conquer the known disadvantages of the anticancer drugs, for instance, the low bioavailability and high cytotoxic side effects (Gupta et al., 2009; Kim et al., 2010). In one case, a novel nanogel-drug conjugates based on membranotropic cholesteryl-HA (CHA) has been synthesized for efficient targeting and suppression of drugresistant tumors. These conjugates could significantly increase the bioavailability of poorly soluble drugs with previously reported activity against CSC, such as etoposide, salinomycin, and curcumin. The resultant CHA-drug nanogels demonstrated higher cytotoxicity in CD44-expressing drug-resistant human breast and pancreatic adenocarcinoma cells compared to that of free drugs and non-modified HA-drug conjugates. Approaches using polymeric nanoparticles to encapsulate drugs for CSC targeting attract more attentions in cancer treatment because of the advantages including the controllable release of drugs, structural design for targeting, and functional design for diagnosis (Wei et al., 2013).

In this review, we first introduce the specific characteristics of CSCs and the common strategies for CSCs targeting. We then outline the current polymeric nanoparticles used for CSCs targeting in the application of cancer therapy.

\section{CURRENT CANCER THERAPY VIA CSCS INHIBITION}

With the recent exploration advancing in CSCs, the CSCs targeted therapy has brought a new hope to the cancer patients. The identification and characterization of CSCs have revealed numerous strategies of cancer treatment in-depth via specific molecular therapies (Visvader and Lindeman, 2008): for example, interfering the cell growth microenvironment of a tumor; targeting the specific biomarkers of CSCs; or inhibiting the key signaling pathways to interfere CSCs activity. Strategies of CSCs targeting based on their properties were showed in Figure 1.

\section{Targeting the Tumor Microenvironment}

A niche is the CSCs intrinsic property built by tumor microenvironment to prevent from apoptosis by chemotherapy. The CSCs niche is a dynamic supportive system with specific anatomic and functional features to modulate how the cells take part in the process of tumor growth and metabolism (Scadden, 2006).

Folkins et al. (2007) reported that instead of targeting the CSCs directly, attacking the vascular niche to collapse the resistance ability of tumor against the chemotherapy. In another case, Gulotta et al. (2007) suggested that in order to destroy the drugresistant brain tumor, to attack the key member of CSCs nicheendothelial cells indirectly would be a promising strategy. The anti-inflammatory drugs (IFN- $\beta$ ) could also serve as potential components of CSC-focused therapies to disrupt the vascular niche of glioma CSCs (Williams et al., 2010).

Whereas neither antiangiogenic nor tumor cell cytotoxic effects alone are sufficient to reduce the CSCs fraction, therapies combining these effects are capable of selectively eliminating CSCs from the tumor. The possible reason for these observations is that antiangiogenic therapies disrupt a glioma CSCs vascular niche. The subsequent loss in communication between CSCs and their niche would elicit a reduction or loss of certain stem cell characteristics, which could include aspects of stem cell-associated drug resistance, and may result in increased proliferation rate and reduced DNA repair capacity in CSCs. Such changes would sensitize the CSCs to the cytotoxic effects of cyclophosphamide, therefore allowing combinatorial therapies to selectively eliminate CSCs.

\section{Inhibition of CSCs-Dependent Signaling Pathways}

The maintenance of stem cell nature partly depends on the regulation of relative signaling pathways. Several signaling pathways have been implicated in CSC chemoresistance in a variety of cancers. The pathways and signal molecules related to the control of CSCs self-renewal, differentiation and apoptosis include PI3K/Akt, PTEN, JAK/STAT, Wnt/ $\beta$-catenin, hedgehog, Notch, and NF- $\kappa$ B etc. as shown in Figure 2 (Gulotta et al., 2007; Baud and Karin, 2009; Burger and Peled, 2009; Chen et al., 2009; Guo et al., 2010; Lorenzo et al., 2010; Roy et al., 2010; Murphy and Stordal, 2011).

The activation of signal molecule Akt plays a critical role for cellular transformation and tumorigenesis. The PI3K/Akt signaling pathway regulated the growth of numerous cancers. Guo et al.'s (2010) study showed that Akt1 improved the resistance to apoptosis by increasing the expression level of Bcl-2 and the phosphorylation of the pro-apoptotic protein $\mathrm{BAD}$. The tumor generation related signaling pathway JAK/STAT was activated at the same time. To control the suppressor of cytokine signaling-3 (SCS-3) is also viewed as an effective method to regulate tumor formation by the activating JAK/STAT signaling pathway (Pradhan et al., 2007). The Notch signaling pathways have also been shown to be involved in tumor development, metastatic initiation and self-renewal processes. The over expression of Notch3 could lead to the expansion 


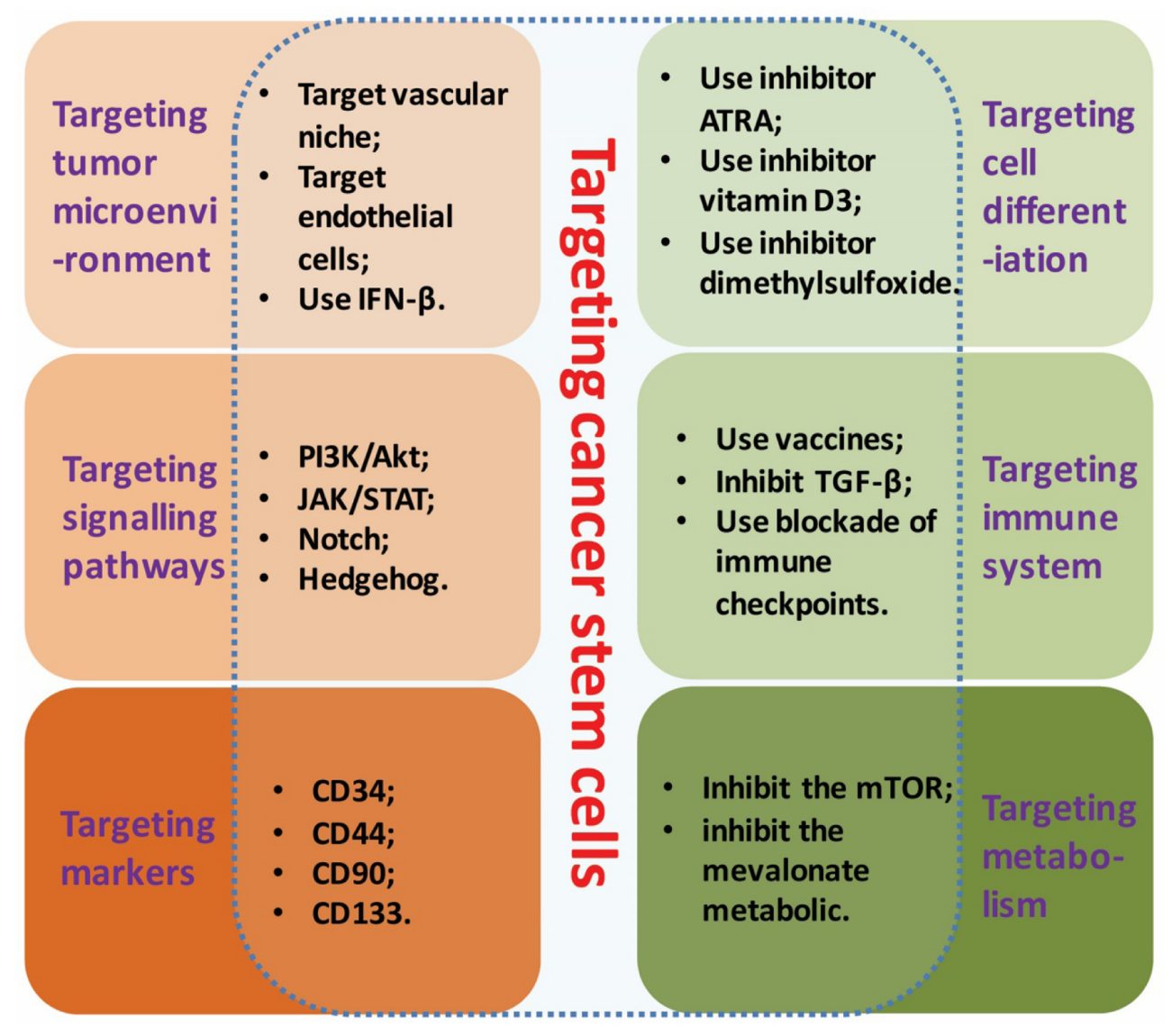

FIGURE 1 | Strategies of CSCs targeting. Strategies of CSCs targeting based on its specific molecular properties include: interfering the growth microenvironment, targeting the biomarkers, inhibiting the key signaling pathways, targeting cell differentiation, targeting immune system and targeting metabolism.

of CSCs and chemoresistance enhancement in ovarian cancer. The sensitivity of CSC was obvious increased to platinum therapy after inhibiting the Notch signaling pathway via siRNA knockout. In addition, the well-known hedgehog and Wnt signaling pathways also play fundamental roles in maintaining CSC populations (Dontu et al., 2011).

\section{Targeting CSC-Dependent Markers}

With the recent advances and growing knowledge on unique CSC biomarkers, tumor targeted therapy has shed new light to the cancer patients. The expressions of CSC surface markers, however, are heterogenous, which are different from patients with the same cancer and the same tumor in different progress stages. Thus, it's critical to evaluate the CSC markers in the tumor of every stage to pick up the CSC relative treatment. Furthermore, the CSC surface marker proteins play important roles not only in cell isolation, identification and observation, but also in the applications of both preclinical diagnosis and clinical therapy (Visvader and Lindeman, 2008).

These markers include CD34, CD44, CD90, CD133, and EpCAM, etc. (Medema, 2013). The ligands or antibodies against tumor surface makers have been widely employed to enhance the specificity of therapeutic strategies. The anti-bodies of these CSCs biomarkers were developed and utilized in several drug delivery systems for tumor targeting and cancer therapy. BourseauGuilmain et al. (2011) prepared lipoprotein-like nanocapsules to deliver AC133 to effectively target CD133 positive CSCs. A CD44-specific tumor-targeting drug was carried by HA for the cancer therapy (Marhaba et al., 2008). An anti-CD90 antibody was also proved to be able to stop the bone marrowderived multipotent stromal cells to differentiate into chondro-, osteogenic, or adipo-lineages (Gundlach et al., 2011).

\section{Interfering the CSCs Differentiation}

Although the anticancer drugs for ideal targeting have being looked for decades, only few are successfully applied in clinical trial. One of them is all-trans retinoic acid (ATRA), Warrell et al. (1993) found that the differentiation of leukemic promyelocytes were blocked and failed to differentiate into mature granulocytes after treating the patients with ATRA. The rational theory of ATRA therapy is ATRA could drive the CSCs to differentiate from stem cell into non-CSCs (e.g., epithelial cells). This case indicated the therapy of interfering CSCs differentiation may be an effective way to treat other forms of cancers. Other agents such as phorbol myristate acetate (Carey et al., 1996), hexamethylamine bisacetamide (Wu et al., 1991), dimethylsulfoxide (Arcangeli 


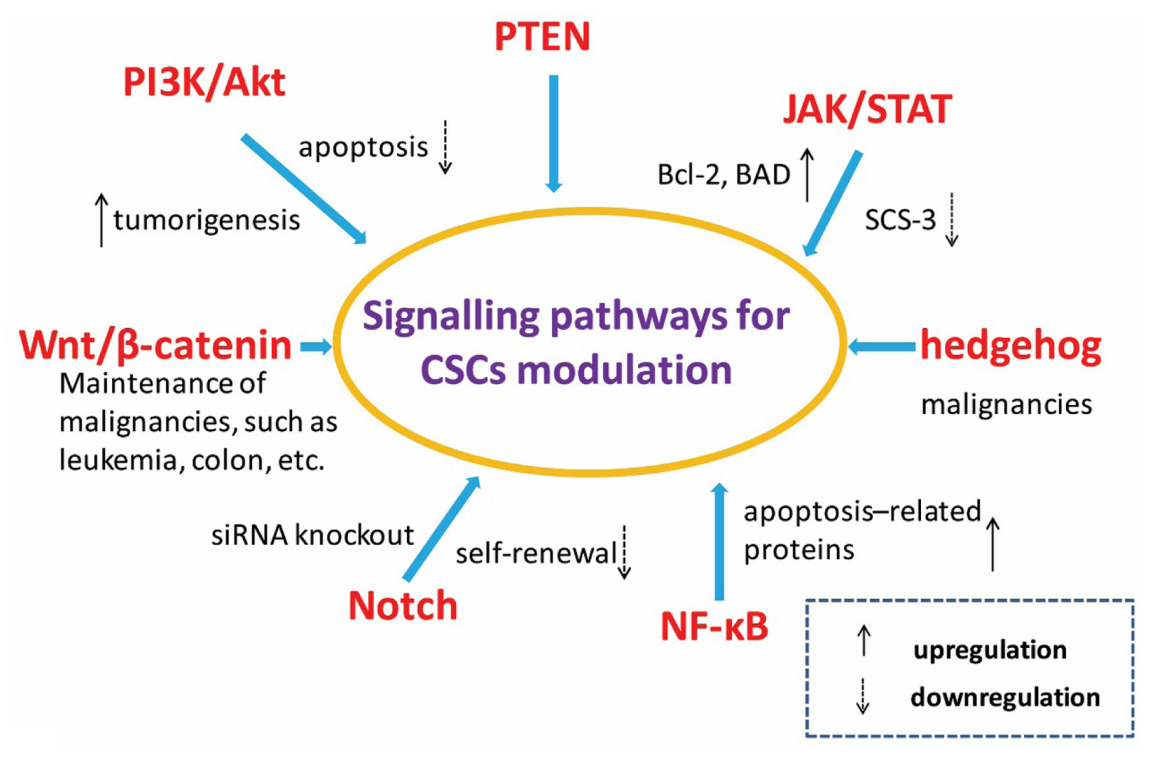

FIGURE 2 | Signal pathways related with cancer stem cells. Pathways and elements involved in the control of selfrenewing and differentiation of cancer stem cells as well as normal stem cells include PI3K/Akt, JAK/STAT, Wnt/ $\beta$-catenin, hedgehog, Notch, NF-kB, and so on.

et al., 1993) and vitamin D3 (Olsson et al., 1984) also were reported to have the similar function in CSCs differentiation therapy.

\section{Immunotherapy against CSCs}

Challenges in CSCs targeting remain intractable issues need to be faced in a long term, while immunotherapy becomes a novel potential interest in the field of cancer therapy. Numerous researches have shown that CSCs can be eradicated by innate immune effector cells. The usage of tumor antigens to directly break the cancer patient's immune system has been explored decades for cancer therapy (Pardoll, 2012). The strategies include: (a) using the specific peptides derived from tumor antigens for cancer vaccination; (b) stimulating the immune cells with dendritic-cells to fight against specific antigens; and (c) interfering the immune responses of anti-tumor via retardant.

The CSCs-secreted TGF- $\beta$ that has additional immunosuppressive effects on other subclasses of $\mathrm{T}$ lymphocytes, was used to avoid being attacked by various cellular components of the immune system (Pardoll, 2012). Castriconi et al. (2009) indicated that the allogeneic and autologous-activated natural killer cells were capable of influencing the stem-like cells to lysis. Todaro et al. (2009) research demonstrated that $\gamma \delta$ T lymphocytes can be used to kill human colon CSCs in vitro. Contag et al. (2010) reported that the oncolytic virus can be delivered into lymphoma cancer stem like cells as vehicles via cytokine-induced killer cells.

\section{Exploiting Metabolic Differences to Target CSCs}

The behaviors of CSCs in the niche are affected by the metabolic products, the CSCs therefor can respond to the conditional environment. Because of the fresh discoveries in the Warburg effect, cancer metabolism has been coming back into spotlight the recent 10 years. This presents that cancer cells generate ATP through glycolysis (even under non-hypoxic conditions) rather than oxidative phosphorylation (Warburg et al., 1952). Another research reported that they set up a mouse model of lung CSCs and proved repressive functions of LKB1 on metastasis through regulation of NEDD9 and found that LKB1 mutation correlates with lung CSCs. These findings establish that LKB1 suppresses lung tumorigenesis through at least three independent mechanisms influencing tumor initiation, differentiation, and metastasis (Ji et al., 2007). With the development of novel therapeutics based on the continuous studies on signaling regulating metabolic pathways, the role of cell metabolism is shedding new light in cancer research. For instance, the metabolism of cancer cells can be changed via inhibiting the mTOR signaling by hypoxia (Wouters and Koritzinsky, 2008). Inhibiting the mevalonate metabolic pathway which plays vital role in breast CSCs generation by hydroxy-3-methylglutaryl CoA reductase blockers significantly break the CSCs growth process (Ginestier et al., 2012). These cases indicated that tuning the CSC metabolism could be considered as an effective way to interfere the CSCs activities by specific targeting and kill the CSC cells from the normal cells.

The CSCs extend our horizons of knowledge on cancer and provide a new approach to eradicate malignancies. It is proved that CSCs exist in various malignancies and play critical role in tumor initiation and development. While the conventional cancer therapy ways which are effective in various cancer treatments, have no more obvious effect in chemo- and radioresistant cancer stem cells. Thus, a wide variety of CSCs targeting strategies are being investigated for the treatment of malignant tumors. What's more, the unique characteristics of CSCs remain needed to be further explored to promote the understanding and 
utilization of CSCs. The strategies mentioned above are just a part of ways effectively used in CSCs targeting therapy. Although promising, the challenges of specificity and potential toxicities still need to be further tackled before the clinical trials. The drugs used for CSCs targeting based on their characteristics may have some adverse effects on the maintenance of normal cells or organs. Therefore, it will be critically important to develop new therapies that exclusively target CSCs and avoid targeting normal stem cells or cells in the future.

\section{POLYMERIC DRUG-LOADED NANOPARTICLE DELIVERY SYSTEM}

Although the huge progress emerges in medical technique, the cancer therapy remains a challenging because of the low cure rate. The polymeric drug-loaded nanoparticles have been viewed as a novel promising strategy for cancer treatment because they not only can improve the drug pharmacokinetics but also further response to the permeation and retention (EPR) effect to enhance the accumulation of drugs at the site of the tumor during cancer treatment (Kapoor et al., 2015).

There are lots of factors should be included in an ideal design of polymeric nanoparticles system for drug delivery, including biocompatibility, particle size, drug loading rate, and pharmacokinetics, etc. (Vinogradov and Wei, 2012). The nanoparticles make themselves better cell affinity and easier uptake by the target cells through interactions ligands on the nanoparticles' surface with receptors on the cell membranes. There are numerous advantages of nanoparticles using for anti-cancer drugs delivery in cancer treatment: (a) Increase the drug circulation half-lives: Polyethylene glycol, polyvinyl alcohol, polysaccharides, and surfactants such as poloxamer were used to decorate the surface of nanovehicles to circumvent the opsonization and the reticuloendothelial system. Cargos in these nanovehicles turned into decreased apparent clearance, extended apparent circulation half-life, as well as lessen apparent volume of distribution, eventually producing prolonged drug retention time in the circulation system. (b) Improve the drug aqueous solubility and stability: nanotechnologies are broadly drawing attention as favorable option for oral administration of drugs with poor oral bioavailability. Besides increasing drug uptake via cellmediated active absorption (Florence, 2005), drug encapsulated nanoparticles could diminish transporter-mediated efflux (Ling et al., 2010). Additionally, drug loaded in nanoparticles protects the drug from degrading by harsh conditions in the GI system (Italia et al., 2007; Mittal and Kumar, 2009) and decreases hepatic first-pass metabolism (Anand et al., 2007; Mittal et al., 2007). Drug loaded in nanoparticles could safely go through the gastrointestinal tract and liver avoiding degradation/metabolism, which would result in reduction of the metabolic clearance and increasing of its absorption and oral bioavailability as well. (c) Control the drug release and specific targeting: some of the most amazing advantages of nanocarriers include their tunable payload release and the ability to specifically target their payload to diseased tissues and cells by modification of their surface chemistries, and finally their ability to respond to various internal and external stimuli for "triggered" release to achieve temporal and spatial control over the release of therapeutic payloads. (d) Enhance the drug bioavailability: nanotechnology is increasingly considered to be the technology of the future. Among the wide applications of nanotechnology is the use of nanoparticles for enhancing the bioavailability. Poorly water-soluble drug candidates encountered in drug discovery cause increasing problems of poor and variable bioavailability. It was suggested that nanoparticles could enhance the oral drug bioavailability probably because the special stability (thermodynamic and kinetic stability) facilitate the safe transport of drugs through the G1 tract and/or the mucoadhesivity of nanoparticles prolonging the residence time in the gut, and the P-gp inhibitors contributing to drug accumulation.

\section{Designs of Nanoparticles for Drug Delivery System}

Although the traditional cancer therapy techniques are improving and various novel therapeutic strategies are being developed, polymeric nanoparticles are still viewed as the first option for drug delivery and also widely used in the research of other diseases due to their excellent physicochemical properties (Fonseca et al., 2013, 2014), such as particle size, surface charge, surface chemistry, hydrophobicity, degree of rigidity, and degradation speed. The polymeric nanoparticles also can be decorated on the surface to obtain the appropriate functions to fit the tumor environment or properties of drugs. The usual modification includes surface charge modification, bioactive peptides graft, amphipathic compound graft and siRNA (Figure 3).

Surface modification of polymeric nanoparticles has been proved to be useful in offering multi-functionality, and cannot only easily optimize the nanoparticles distribution in cells and tissues, but also promote cellular uptake or reduce cellular interactions. The modified polymeric nanoparticles can accumulate the loaded-drugs within the tumor tissues accurately and avoid damaging the nearby healthy tissues.

\section{Particle Size}

One of the most widely utilized mechanisms of drug delivery system for targeting is based on particle size. The enhanced EPR effect allows nanoparticles less than $500 \mathrm{~nm}$ to penetrate tumor vasculature and gather in the regions of tumor (Petros and Desimone, 2010). The larger nanoparticles would be prevented getting there because they are trapped in the lungs or removed out the body by the reticuloendothelial system (RES). The nanoparticles are usually modified to obtain neutral surface charge by coating hydrophilic molecules such as PEG to eliminate particle aggregation and avoid RES for the EPR effect (Torchilin, 2011).

\section{Transferrin}

Transferrin (Tf) is a glycoprotein used to improve targeting efficiency for the tumor cells which overexpress the Tf-receptor by grafting to nanoparticles made of poly(L-lysine) (Wagner et al., 1990), polyethyleneimine (Ogris et al., 2003), cyclodextrin (Bellocq et al., 2003), or polyamidoamine (Huang et al., 2007). 


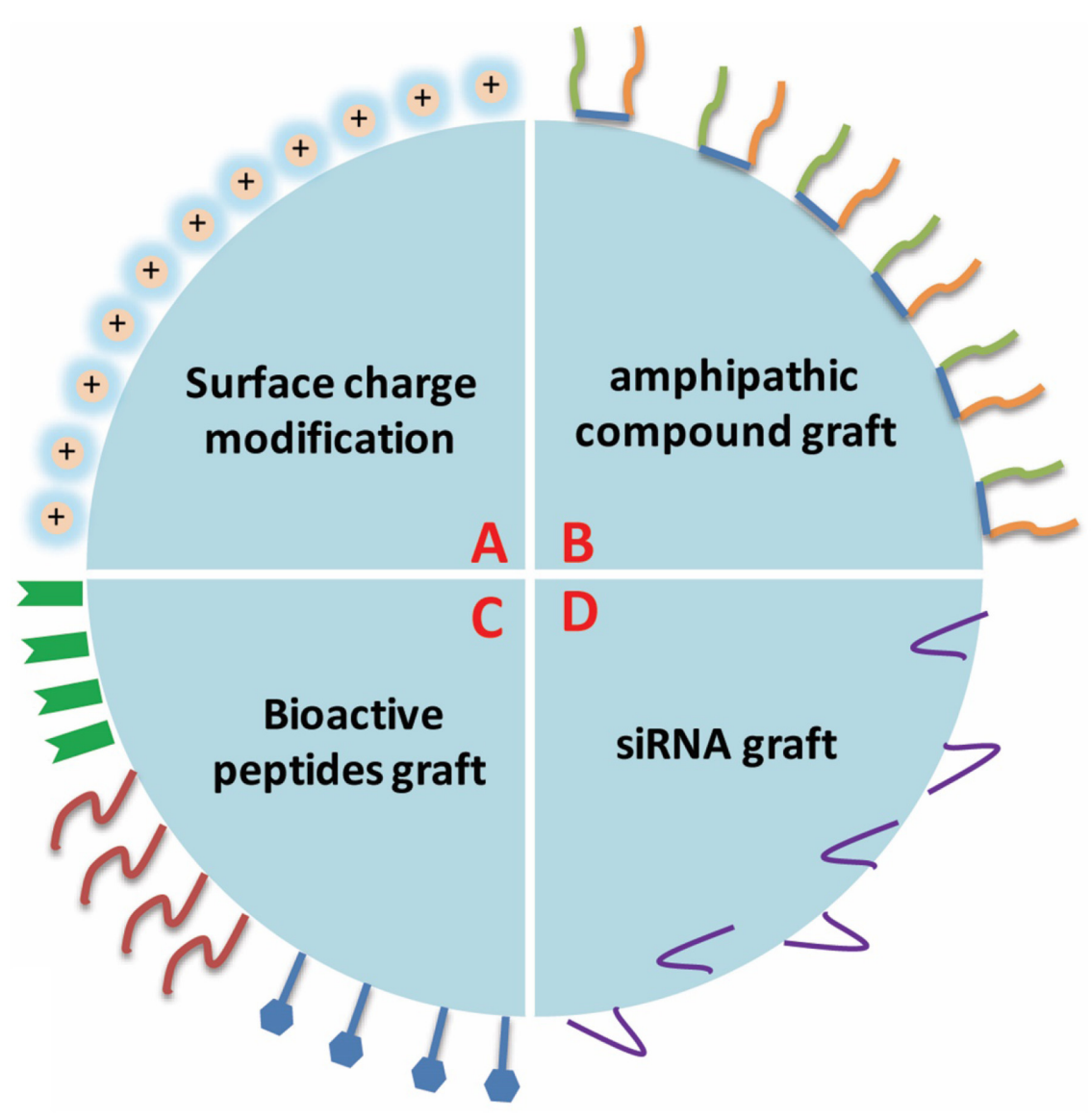

FIGURE 3 | The usual surface modifications of polymeric nanoparticles. The usual modification includes surface charge modification, bioactive peptides graft, amphipathic compound graft, and siRNA, etc.

Tf was reported to be conjugated onto the surface of PEI and PEI-PEG nanoparticles for plasmid delivery, which showed the clear improvement of tumor targeting and reduction on offtarget transfection (Hildebrandt et al., 2003). Tf conjugated to cyclodextrin-PEG nanoparticles (CALAA-01) for the delivery of small interfering RNA (siRNA) to solid tumors is undergoing FDA Phase I clinical trials (Davis, 2009).

\section{Epidermal Growth Factor Receptor}

The epidermal growth factor receptor (EGFR), which considered as a signal of poor prognosis, overexpressed in approximately $30 \%$ of solid tumors during cancer therapy (Jones et al., 2006). The EGFR targeted polymeric nanoparticles were shown to improve tumor specific transfection compared to non-targeted nanoparticles (Kathrin et al., 2011).

\section{RGD Peptide}

The RGD peptide (arginine, glycine, aspartic acid) is reported to strongly target the $\alpha \mathrm{v} \beta 3$ integrin receptor which is selectively expressed in tumor vasculature to enhance the cellular uptake, and weakly bind to some other integrin receptors (Kunath et al., 2003). PBAE nanoparticles attached by RGD peptide for the targeted delivery of DNA to tumor cells was proved to improve transfection of cells overexpressing integrin receptors (Green et al., 2007). Both the RGD peptide and cyclic-RGD peptide conjugated to nanoparticles for targeted delivery of plasmids to tumor cells has also been shown to improve transfection in vivo for $24 \mathrm{~h}$ after IV administration in mice (Sakae et al., 2008).

\section{Antibodies}

Antibodies can be employed to specifically target a tumor that expresses a tumor specific antigen. PEI conjugated with HER2 antibodies for DNA delivery improved transfection against $\mathrm{HER}^{+}$breast cancer cell lines in vitro (Chiu et al., 2004). PEI/DNA nanoparticles conjugated with antibodies of prostate specific membrane antigen for targeting prostate cancer cells in vivo was reported to have a 20 times enhancement compared to non-targeted nanoparticles (Moffatt et al., 2006).

In additionally, Folate (Zhang et al., 2010), HA (Needham et al., 2012), and some specific carbohydrates have been also employed as targeting moieties for appropriate polymeric nanoparticle delivery to CSCs.

The increasing attentions on approaches of cancer treatments have raised the research interest of CSCs targeting via polymeric nanoparticle. The use of ligands conjugated to the surface of nanoparticles can allow the drugs enter CSCs and regulate lots of 
genes or protein expression, kill the CSCs eventually. To improve the transfection efficiency and the capacity of cellar uptake, the factors such as ligand density, surface charge and affinity have to be considered in the process of material design.

\section{Applications of Polymeric Nanoparticles for CSCs Targeting Therapy}

Polymeric nanoparticles have been regarded as the most efficient carriers for drug delivery due to their excellent pharmacokinetic properties such as drug loading, drug release, structure stability and nanoparticles degradation (Alexis et al., 2010; Pérez-Herrero and Fernández-Medarde, 2015). The recent research hotspot of polymers utilized for drug loaded nanoparticles include poly (D, L-lactic-co-glycolic acid) (PLGA), polylactic acid (PLA), poly (ethylene glycol) (PEG), chitosan (CS), and hyaluronic acid (HA), etc.

\section{Poly (lactide-co-glycolide)}

Poly (lactide-co-glycolide) (PLGA) is one of the few US FDA approved polymers for clinical applications due to its outstanding properties such as biodegradability and biocompatibility (Chaubal, 2002; Astete and Sabliov, 2006). So far, it's the most widely used synthetic polymers in drug-loaded nanoparticles development for cancer therapy (Biondi et al., 2008). Ni et al. (2015) also reported that the CD133 grafted PLGA nanoparticles loaded with salinomycin were capable to target the CD133 osteosarcoma CSCs.

To kill both the normal cancer cells and CSCs, salinomycin (SLM) and paclitaxel (PTX) were embedded by PGLA for CD44 targeted chemotherapy with emulsion solvent diffusion method. This combinational therapy demonstrated the synergistic effects of dramatically reducing the $\mathrm{CD} 44^{+}$cells (Muntimadugu et al., 2016). In order to eliminate drug resistance and relapse of breast CSCs, Swaminathan et al. (2013) prepared PLGA nanoparticles to target CD133 via conjugating an anti-CD133 monoclonal antibody. The result showed that the breast CSCs population was significantly reduced and the therapeutic efficacy was obviously enhanced. To further actively target CSCs to reduce their drug resistance and stabilizing agent, HA and PF127 were conjugated onto PLGA to get the smart nanoparticles for cancer therapy. These nanoparticles were synthesized using the double-emulsion approach and allowing for acidic $\mathrm{pH}$-triggered drug release and thermal responsiveness at the same time (Wang et al., 2015).

\section{Hyaluronic Acid}

Hyaluronic acid (HA) is an anionic, non-sulfated glycosaminoglycan distributed in connective, epithelial and neural tissues. HA has been widely studied in cancer treatment by conjugating different drugs due to its outstanding characteristics including biocompatibility, biodegradability, and non-immunogenicity (Luo et al., 2000; Prestwich, 2011). Many drug-resistant cancer cells and CSCs were reported to enhance the expression levels of some biomarker receptors. These markers conjugated onto HA can use to target CSCs for the cancer therapy. For instance, cholesterol-modified HA (CHA) conjugated with selected drugs that are highly active against CSCs via biodegradable covalent linkage can be used to deliver the drug efficiently for drug-resistant cancer. Banzato et al. (2008) developed a paclitaxel-hyaluronan bioconjugate (ONCOFID ${ }^{\mathrm{TM}}-\mathrm{P}, 20 \%$ drug content) to target human ovarian cancer IGROV-1 and OVCAR-3 xenografts via the intraperitoneal administration. The HA was also structurally functionalized for siRNAs encapsulation to transfect siRNAs into cancer cells that overexpress CD44 receptors. Several HA based drug-loaded nanoparticles delivery systems have been delivered into the cancer cells overexpressed the CD44 receptors, and some decorated HA derivatives can effectively silence the related gene activity in vitro and in vivo (Choi et al., 2009).

However, there is a defect of HA-based drug delivery nanoparticles in cancer therapy application. The delivery system preferentially accumulates in the liver after systemic administration. To address this issue, poly (ethylene glycol) is employed to conjugate onto HA. The result indicated that the PEG-modified HA-nanoparticles were significantly reduced in the liver, and lengthened the times stay in blood circulation, eventually enhanced the veracity of CSCs targeting (Choi et al., 2011).

\section{Poly (ethylene glycol)}

Poly (ethylene glycol) (PEG) is a hydrophilic polymer widespread use in the field of biomedicine due to its excellent water solubility, chain mobility, non-toxicity, and non-immunogenicity. In order to solve the low water solubility and unstable properties of bortezomib in clinical application, the amphiphilic copolymer poly (ethylene glycol)-block-poly(d,l-lactide) (PEG-b-PLA) nanoparticles were engineered. Their study demonstrated that the bortezomib loaded in PEG-b-PLA nanoparticles can enter into the targeted CSCs, and induce the CSCs apoptosis and death. This DDS was proved to keep the drugs in CSCs for a long time and accumulate the drugs in lesion tissue, and eventually inhibit the proliferation of CSCs, promote the therapeutic efficacy in breast cancer therapy (Shen et al., 2015). To lower the toxicity and improve the drug release, the diblock copolymer nanoparticles of poly (ethylene glycol) (PEG) and functional polycarbonates (PAC) were fabricated via metal-free organocatalytic ring-opening polymerization to provide the narrow size distribution and high loading capacity for anticancer drugs. Doxorubicin (DOX)-loaded PEG/PAC nanoparticles showed higher antitumor efficacy and decreased toxicity than free DOX in vivo (Ke et al., 2014). To increase the solubility, the potent vascular-disrupting agent was bonded to a PEG-based polymer to achieve self-assemble in the aqueous solution. DOX then was loaded into these nanoparticle carriers to improve the cytotoxicity and loading capacity. The in vivo study on MCF-7 tumor bearing nude mice demonstrated that the antitumor efficiency was significantly improved (Vinogradov and Wei, 2012). To make the PEG nanoparticles intelligent, Liu et al. (2013) developed the gelatinases-stimuli poly (ethylene glycol)-peptide-poly( $\varepsilon$-caprolactone) drugs delivery system. The nanoparticles were developed to investigate the targeting effects on CSCs and non-stem cancer cells by carrying both miR-200c and docetaxel (DOC). The results demonstrated that the tumors growth speed was decreased after the miR$200 \mathrm{c} / \mathrm{DOC}$ nanoparticles were put into the nude mice. The 
PEG-based gelatinases-response drug-loaded nanoparticles may also suggest that the loading of both drugs and siRNA can target not only CSCs but also non-stem cancer cells at the same time.

\section{Polylactic Acid}

polylactic acid (PLA) is FDA approved biodegradable polymer and fumaric acid existed in the Kreb cycle. It can be totally excreted through metabolism after degradation. Yang et al. (2015) established lung cancer stem-like cells (CSLCs)-targeting encapsulated docetaxel PLA nanoparticles for anti-metastatic therapy. The in vitro and in vivo experimental results confirmed the outstanding anti-metastatic performance of CSLC-targeting DTX nanoparticle. Liang et al. (2015) also have the similar study, they fabricated the PLA nanoparticles loading DTX based on the emulsion solvent evaporation method. Their study indicated that the tumor growth inhibition and anti-metastatic efficacy in the SCLC targeting drug delivery system for cancer treatment. Pandey et al. (2015) developed PLA nanoparticles to deliver quercetin $(\mathrm{Qt})$ to inhibit the human breast cancer cells. Results showed that the PLA-Qt nanoparticles displayed excellent sustained release kinetics and can be used as a novel vehicle for the cancer treatment. In another case, the cellulose, that was reported to protect drugs and deliver them to the target site, was grafted on poly (L-lactic acid) (PLLA) to load betulinic acid for anti-cancer therapy. Results demonstrated that the experimental effect in the CEg-PLLA/BA nanoparticles group showed not only fewer side effects but also better efficacy in tumor proliferation suppression than that of BA alone (Dai et al., 2014). To regulate the signal molecules related to breast CSCs, the MPEG-b-PLA nanoparticles were developed to deliver the small molecules (Ahmed and Discher, 2004; Blanco et al., 2007). Low-dose of DAC encapsulation can decrease the number of CSCs in mammospheres of MDA-MB-231 cells and rectify their sensitivity to DOX. While systemic delivery of DAC cannot only obviously decrease the expression level of DNMT1 and DNMT3b in a MB-MDA-231 xenograft murine model, but also up-regulate the caspase- 9 expression to enhance the sensitivity of CSCs to DOX. The controllable MPEG-b-PLA nanoparticles loading capacity offers a promising strategy for breast cancer therapy ( $\mathrm{Li}$ et al., 2014).

\section{Chitosan}

Chitosan, derived from chitin, is a positive biocompatible and biodegradable natural polysaccharide. It is widely used in the field of biomedicine including drug and gene delivery, tissue engineering, wound healing, and antimicrobial (Senel and Mcclure, 2004; Riva et al., 2011). The chitosan nanoparticles were developed for targeting the overexpressed CD44 receptors on CSLCs. The nanoparticles around $20 \mathrm{~nm}$ were delivered into the tumor to actively target and be internalized in CD44 ${ }^{+}$CSLCs by EPR effect. The drugs in nanoparticles then would release out and diffuse into the cytosol, eventually accumulate in the nuclei to produce cytotoxicity to kill CSLCs eventually (Chauhan et al., 2012; Sykes et al., 2014). To lower the toxicity of platinate, Nascimento et al. (2015) developed the chitosan-based polymeric nanoparticles to encapsulate hydrophobic drugs by grafting
PEG and peptides. Their study reported that the modified platinate encapsulated into chitosan-based nanoparticles can obviously decreased the toxicity in cells. The chitosan also can be used as cell recognition. A doxorubicin-encapsulated polymeric nanoparticles decorated with chitosan on the surface were developed to specifically target the CD44 receptors of the cancer treatment. This nanoparticle system released the doxorubicin in acidic environments localized in the cellular endosomes/lysosomes to achieve the goal of killing the CSCs (Rao et al., 2015).

In summary, a large number of polymeric nanoparticles with different physicochemical characteristics for multiple drugs loading have been developed to control the drugs release via responding internal or external stimuli to significantly improve therapeutic efficacy by CSCs targeting.

\section{CONCLUSION AND PROSPECTS}

Cancer is one of the most complicated diseases. Although the drug-loaded nanoparticles have improved preclinical and clinical outcomes for cancer treatment, the therapeutic efficacy is still limited due to the genetic diversity and the biological complexity of tumor. Therefore, modifying the chemical or physical characteristics of polymeric nanoparticles to meet the need of different drugs with different physicochemical characteristics is an attractive strategy for clinical CSCs targeting.

In this review, we introduce the ways of CSCs inhibition based on the CSCs profiles. Then we enumerate several representative decorated polymeric nanoparticles for drugs delivery in the applications of cancer therapy.

More and more studies have confirmed the CSCs is critical factor for tumor resilience, resisting to radiotherapy, and were accused for tumor Resurrection. As that knowledge about CSC advanced, cutting-edge ideas and technologies have kept coming forth in order to eliminate malignancies. Several kinds of carcinomas may harbor CSCs with mesenchymal properties, and self-renewal and multi-differentiation properties, while it's hard to find an exclusive selection marker to isolate and target the corresponding CSCs. Fractions of CSCs could be masked by a tumor with heterogeneousness, that indicate targeting treatment for CSC isn't a simple task. Therefore, many different targeted therapies for systemic treatment for CSCs have been put forward recently. The studies mainly focused on inhibition of the VEGF and mTOR pathways and targeted on markers (such as CD105 and CXCR4) of CSCs. Although various fresh trials have been carried out in clinic, there are still observable restrictions occur in getting to CSCs in vivo due to the unreachability of the deep area of tumor or because of the tumor milieu, these restrictions could be overpowered by nanotechnological strategies. Versatile CSCtargeting approaches together with newly discovered pathway and cytotoxic agents could contribute to tackle those difficulties in CSCs remedy. Accumulating knowledge on CSCs will lead us to comprehensively understand the responses of CSCs to various treatments, different phases of disease, and individual patient. Finally, all of these finding would bring about more patienttailored and targeted treatments. Sensibly built-up nano-therapy 
for CSCs explores new routes for clinical study and brings in new faith in practical use.

In the recent years, the hot research interest mainly focusses on design of novel polymeric nanoparticle-based therapies for CSC targeting in cancer treatment. Polymeric nanoparticles can be expediently modified with the cell-recognizable moieties on their surfaces, and designed in appropriate size and structure for pharmaceutical applications. The polymeric nanoparticle-based approaches have increased the drug safety, controllable drug release, and targeting efficacy, and lowered the immunogenicity and tumorigenesis.

Novel therapeutic strategies for combination therapy and stimuli-activated drug delivery are also the representative of new therapeutic trends. For instance, to deliver two or more drugs simultaneously would improve different tumor growth-related signaling pathways targeting, leading to a synergistic therapy effect.

However, these approaches are still in the early stages of development and have many theoretical and technical limitations, issues of dose-related toxicity and side effects are also needed to be addressed. Furthermore, the innovative in vitro evaluation system has to be improved to accurately predict the in vivo pharmacokinetics of drugs in humans. In spite of small

\section{REFERENCES}

Adams, J. M., and Strasser, A. (2008). Is tumor growth sustained by rare cancer stem cells or dominant clones? Cancer Res. 68, 4018-4021. doi: 10.1158/00085472.CAN-07-6334

Ahmed, F., and Discher, D. E. (2004). Self-porating polymersomes of peg-pla and peg-pcl: hydrolysis-triggered controlled release vesicles. J. Control. Release 96, 37-53. doi: 10.1016/j.jconrel.2003.12.021

Alexis, F., Pridgen, E. M., Langer, R., and Farokhzad, O. C. (2010). Nanoparticle technologies for cancer therapy. Handb. Exp. Pharmacol. 197, 55-86. doi: 10. 1007/978-3-642-00477-3_2

Anand, P., Kunnumakkara, A. B., Newman, R. A., and Aggarwal, B. B. (2007). Bioavailability of curcumin: problems, and promises. Mol. pharm. 4, 807-818. doi: $10.1021 / \mathrm{mp} 700113 \mathrm{r}$

Arcangeli, A., Carlà, M., Del Bene, M. R., Becchetti, A., Wanke, E., and Olivotto, M. (1993). Polar/apolar compounds induce leukemia cell differentiation by modulating cell-surface potential. Proc. Natl. Acad. Sci. U.S.A. 90, 5858-5862. doi: $10.1073 /$ pnas.90.12.5858

Astete, C. E., and Sabliov, C. M. (2006). Synthesis and characterization of plga nanoparticles. J. Biomater. Sci. Polym. Ed. 17, 247-289. doi: 10.1163/ 156856206775997322

Banzato, A., Bobisse, S., Rondina, M., Renier, D., Bettella, F., Esposito, G., et al. (2008). A paclitaxel-hyaluronan bioconjugate targeting ovarian cancer affords a potent in vivo therapeutic activity. Clin. Cancer Res. 14, 3598-3606. doi: 10.1158/1078-0432.CCR-07-2019

Baud, V., and Karin, M. (2009). Is nf-кb a good target for cancer therapy? hopes and pitfalls. Nat. Rev. Drug Discov. 8, 33-40. doi: 10.1038/nrd2781

Beck, B., and Blanpain, C. (2013). Unravelling cancer stem cell potential. Nat. Rev. Cancer 13, 727-738. doi: 10.1038/nrc3597

Bellocq, N. C., Pun, S. H., Jensen, G. S., and Davis, M. E. (2003). Transferrincontaining, cyclodextrin polymer-based particles for tumor-targeted gene delivery. Bioconjug. Chem. 14, 1122-1132. doi: 10.1021/bc034125f

Biondi, M., Ungaro, F., Quaglia, F., and Netti, P. A. (2008). Controlled drug delivery in tissue engineering. Adv. Drug Deliv. Rev. 60, 229-242. doi: 10.1016/ j.addr.2007.08.038

Blanco, E., Bey, E. A., Dong, Y., Weinberg, B. D., Sutton, D. M., Boothman, D. A., et al. (2007). $\beta$-lapachone-containing peg-pla polymer micelles as novel animal models have been used to evaluate the therapeutic effect, it is far away from a perfect predictor of the drug efficacy in humans.

The revolutionary developments of novel drugs and new drug delivery systems are urgently needed, and the in vitro models and in vivo pharmacokinetic profiles also need to be improved to enhance the CSCs targeting for the cancer therapeutic effect.

\section{AUTHOR CONTRIBUTIONS}

BL summarized the literature, wrote the manuscript and drew the figures. QL wrote the manuscript and revised the manuscript. HD and JM supervised all the works and provided critical comments.

\section{ACKNOWLEDGMENTS}

This work was supported by the National Key Research and Development Plan of China (No.2016YFC1101605), the Major Program of National Natural Science Foundation of China (No.81190133), and the National Science Foundation of China (No.31460256, No.81360488).

nanotherapeutics against nqo1-overexpressing tumor cells. J Control. Release 122, 365-374. doi: 10.1016/j.jconrel.2007.04.014

Bonnet, D., and Dick, J. E. (1997). Human acute myeloid leukemia is organized as a hierarchy that originates from a primitive hematopoietic cell. Nat. Med. 3, 730-737. doi: 10.1038/nm0797-730

Bourseau-Guilmain, E., Griveau, A., Benoit, J. P., and Garcion, E. (2011). The importance of the stem cell marker prominin-1/cd133 in the uptake of transferrin and in iron metabolism in human colon cancer caco- 2 cells. PLoS ONE 6:e25515. doi: 10.1371/journal.pone.0025515

Burger, J. A., and Peled, A. (2009). Cxcr4 antagonists: targeting the microenvironment in leukemia and other cancers. Leukemia 23, 43-52. doi: 10.1038/leu.2008.299

Carey, J. O., Posekany, K. J., Devente, J. E., Pettit, G. R., and Ways, D. K. (1996). Phorbol ester-stimulated phosphorylation of pu.1: association with leukemic cell growth inhibition. Blood 87, 4316-4324.

Castriconi, R., Daga, A., Dondero, A., Zona, G., Poliani, P. L., Melotti, A., et al. (2009). Nk cells recognize and kill human glioblastoma cells with stem cell-like properties. J. Immunol. 182, 3530-3539. doi: 10.4049/jimmunol.0802845

Chaubal, M. (2002). Polylactides/glycolides-excipients for injectable drug delivery and beyond. Drug Deliv. Technol. 2, 34-36.

Chauhan, V. P., Stylianopoulos, T., Martin, J. D., Popović, Z., Chen, O., Kamoun, W. S., et al. (2012). Normalization of tumour blood vessels improves the delivery of nanomedicines in a size-dependent manner. Nat. Nanotechnol. 7, 383-388. doi: 10.1038/nnano.2012.45

Chen, A. M., Zhang, M., Wei, D., Stueber, D., Taratula, O., Minko, T., et al. (2009). Co-delivery of doxorubicin and bcl-2 sirna by mesoporous silica nanoparticles enhances the efficacy of chemotherapy in multidrug-resistant cancer cells. Small 5, 2673-2677. doi: 10.1002/smll.200900621

Chen, K., Huang, Y. H., and Chen, J. L. (2013). Understanding and targeting cancer stem cells: therapeutic implications and challenges. Acta Pharmacol. Sin. 34, 732-740. doi: 10.1038/aps.2013.27

Chiu, S. J., Ueno, N. T., and Lee, R. J. (2004). Tumor-targeted gene delivery via anti-her2 antibody (trastuzumab, herceptin ${ }^{\circledR}$ ) conjugated polyethylenimine. J. Control. Release 97, 357-369. doi: 10.1016/j.jconrel.2004.03.019

Choi, K. Y., Chung, H., Min, K. H., Hong, Y. Y., Kim, K., Park, J. H., et al. (2009). Self-assembled hyaluronic acid nanoparticles for active tumor targeting. Biomaterials 31, 106-114. doi: 10.1016/j.biomaterials.2009.09.030 
Choi, K. Y., Min, K. H., Yoon, H. Y., Kim, K., Park, J. H., Kwon, I. C., et al. (2011). PEGylation of hyaluronic acid nanoparticles improves tumor targetability in vivo. Biomaterials 32, 1880-1889. doi: 10.1016/j.biomaterials.2010.11.010

Clarke, M. F., Dick, J. E., Dirks, P. B., Eaves, C. J., Jamieson, C. H., Jones, D. L., et al. (2006). Cancer stem cells-perspectives on current status and future directions: aacr workshop on cancer stem cells. Cancer Res. 66, 9339-9344. doi: 10.1158/ 0008-5472.CAN-06-3126

Clevers, H. (2011). The cancer stem cell: premises, promises and challenges. Nat. Med. 17, 313-319. doi: 10.1038/nm.2304

Contag, C. H., Sikorski, R., Negrin, R. S., Schmidt, T., Bachireddy, P., Felsher, D. W., et al. (2010). Definition of an enhanced immune cell therapy in mice that can target stem-like lymphoma cells. Cancer Res. 70, 9837-9845. doi: 10. 1158/0008-5472.CAN-10-2650

Dai, L., Yang, T., He, J., Deng, L., Liu, J., Wang, L., et al. (2014). Cellulosegraft-poly(l-lactic acid) nanoparticles for efficient delivery of anti-cancer drugs. J. Mat. Chem. B 2, 6749-6757. doi: 10.1039/C4TB00956H

Davis, M. E. (2009). The first targeted delivery of sirna in humans via a selfassembling, cyclodextrin polymer-based nanoparticle: from concept to clinic. Mol. Pharm. 6, 659-668. doi: 10.1021/mp900015y

Dean, M., Fojo, T., and Bates, S. (2005). Tumour stem cells and drug resistance. Nat. Rev. Cancer 5, 275-284. doi: 10.1038/nrc1590

Dick, J. E. (2009). Looking ahead in cancer stem cell research. Nat. Biotechnol. 27, 44-46. doi: 10.1038/nbt0109-44

Dontu, G., Jackson, K. W., Mcnicholas, E., Kawamura, M. J., Abdallah, W. M., and Wicha, M. S. (2011). Role of notch signaling in cell-fate determination of human mammary stem/progenitor cells. Breast Cancer Res. 6, 1-11.

El-Serag, H. B. (2001). Epidemiology of hepatocellular carcinoma. Clin. Liver. Dis. 5, 87-107. doi: 10.1016/S1089-3261(05)70155-0

Florence, A. T. (2005). Nanoparticle uptake by the oral route: fulfilling its potential? Drug Discov. Today Technol. 2, 75-81. doi: 10.1016/j.ddtec.2005.05.019

Folkins, C., Man, S., Xu, P., Shaked, Y., Hicklin, D. J., and Kerbel, R. S. (2007). Anticancer therapies combining antiangiogenic and tumor cell cytotoxic effects reduce the tumor stem-like cell fraction in glioma xenograft tumors. Cancer Res. 67, 3560-3564. doi: 10.1158/0008-5472.CAN-06-4238

Fonseca, A. C., Ferreira, P., Cordeiro, R. A., Mendonça, P. V., Góis, J. R., Gil, M. H., et al. (2013). "Drug delivery systems for predictive medicine: polymers as tools for advanced applications," in New Strategies to Advance Pre/Diabetes Care: Integrative Approach by PPPM, ed. M. S. Mozaffari (Dordrecht: Springer).

Fonseca, A. C., Serra, A. C., and Coelho, J. F. J. (2014). Bioabsorbable polymers in cancer therapy: latest developments. EPMA J. 6, 1-18. doi: 10.1186/s13167015-0045-z

Ginestier, C., Monville, F., Wicinski, J., Cabaud, O., Cervera, N., Josselin, E., et al. (2012). Mevalonate metabolism regulates basal breast cancer stem cells and is a potential therapeutic target. Stem Cells 30, 1327-1337. doi: 10.1002/stem.1122

Green, J. J., Chiu, E., Leshchiner, E. S., Shi, J., Langer, R., and Anderson, D. G. (2007). Electrostatic ligand coatings of nanoparticles enable ligand-specific gene delivery to human primary cells. Nano Lett. 7, 874-879. doi: 10.1021/nl062395b

Gulotta, M., Qiu, L., Desamero, R., Rõsgen, J., Bolen, D. W., Callender, R. (2007). Effects of cell volume regulating osmolytes on glycerol 3-phosphate binding to triosephosphate isomerase. Biochemistry 46, 10055-10062. doi: 10.1021/ bi700990d

Gundlach, C. W. IV, Caivano, A., Cabreira-Hansen Mda, G., Gahremanpour, A., Brown, W. S., Zheng, Y., et al. (2011). Synthesis and evaluation of an anti-mlc1 $\times$ anti-cd90 bispecific antibody for targeting and retaining bone-marrowderived multipotent stromal cells in infarcted myocardium. Bioconjug. Chem. 22, 1706-1714. doi: 10.1021/bc200309h

Guo, G., Qiu, X., Wang, S., Chen, Y., Rothman, P. B., Wang, Z., et al. (2010). Oncogenic e17k mutation in the pleckstrin homology domain of aktl promotes v-abl-mediated pre-b-cell transformation and survival of pim-deficient cells. Oncogene 29, 3845-3853. doi: 10.1038/onc.2010.149

Gupta, P. B., Onder, T. T., Jiang, G., Tao, K., Kuperwasser, C., Weinberg, R. A., et al. (2009). Identification of selective inhibitors of cancer stem cells by high-throughput screening. Cell 138, 645-659. doi: 10.1016/j.cell.2009. 06.034

He, L., Gu, J., Lim, L. Y., Yuan, Z., and Mo, J. (2016). Nanomedicine-mediated therapies to target breast cancer stem cells. Front. Pharmacol. 7:313. doi: 10 . 3389/fphar.2016.00313
Hildebrandt, I. J., Iyer, M., Wagner, E., and Gambhir, S. S. (2003). Optical imaging of transferrin targeted PEI| [sol]| DNA complexes in living subjects. Gene Ther. 10, 758-764. doi: 10.1038/sj.gt.3301939

Huang, R. Q., Qu, Y. H., Ke, W. L., Zhu, J. H., Pei, Y. Y., and Jiang, C. (2007). Efficient gene delivery targeted to the brain using a transferrin-conjugated polyethyleneglycol-modified polyamidoamine dendrimer. FASEB J. 21, 11171125. doi: 10.1096/fj.06-7380com

Italia, J. L., Bhatt, D. K., Bhardwaj, V., Tikoo, K., and Kumar, M. N. (2007). PLGA nanoparticles for oral delivery of cyclosporine: nephrotoxicity and pharmacokinetic studies in comparison to Sandimmune Neoral. J. Control. Release 119, 197-206. doi: 10.1016/j.jconrel.2007.02.004

Ji, H., Ramsey, M. R., Hayes, D. N., Fan, C., McNamara, K., Kozlowski, P., et al. (2007). Lkb1 modulates lung cancer differentiation and metastasis. Nature 448, 807-810.

Jones, H. E., Gee, J. M., Hutcheson, I. R., Knowlden, J. M., Barrow, D., and Nicholson, R. I. (2006). Growth factor receptor interplay and resistance in cancer. Endocr. Relat. Cancer 13 (suppl. 1), S45-S51. doi: 10.1677/erc.1.01275

Jordan, C. T., Guzman, M. L., and Noble, M. (2006). Cancer stem cells. N. Engl. J. Med. 355, 1253-1261. doi: 10.1056/NEJMra061808

Kapoor, D. N., Bhatia, A., Kaur, R., Sharma, R., Kaur, G., and Dhawan, S. (2015). Plga: a unique polymer for drug delivery. Ther. Deliv. 6, 41-58. doi: 10.4155/ tde. 14.91

Kathrin, K., Schaffert, D., Willhauck, M. J., Grünwald, G. K., Haase, R., Wunderlich, N., et al. (2011). Epidermal growth factor receptor-targeted 131itherapy of liver cancer following systemic delivery of the sodium iodide symporter gene. Mol. Ther. J. Am. Soc. Gene Ther. 19, 676-685. doi: 10.1038/ mt.2010.296

Ke, X. Y., Ng, V. W. L., Gao, S. J., Tong, Y. W., Hedrick, J. L., and Yang, Y. Y. (2014). Co-delivery of thioridazine and doxorubicin using polymeric micelles for targeting both cancer cells and cancer stem cells. Biomaterials 35, 1096-1108. doi: 10.1016/j.biomaterials.2013.10.049

Kim, S., Shi, Y., Kim, J. Y., Park, K., and Cheng, J. X. (2010). Overcoming the barriers in micellar drug delivery: loading efficiency, in vivo stability, and micelle-cell interaction. Expert Opin. Drug Deliv. 7, 49-62. doi: 10.1517/ 17425240903380446

Kunath, K., von Harpe, A., Fischer, D., Petersen, H., Bickel, U., Voigt, K., et al. (2003). Low-molecular-weight polyethylenimine as a non-viral vector for dna delivery: comparison of physicochemical properties, transfection efficiency and in vivo distribution with high-molecular-weight polyethylenimine. J. Control. Release 89, 113-125. doi: 10.1016/S0168-3659(03)00076-2

Li, S. Y., Sun, R., Wang, H. X., Shen, S., Liu, Y., Du, X. J., et al. (2014). Combination therapy with epigenetic-targeted and chemotherapeutic drugs delivered by nanoparticles to enhance the chemotherapy response and overcome resistance by breast cancer stem cells. J. Control. Release 205, 7-14. doi: 10.1016/j.jconrel. 2014.11.011

Li, Y., Rogoff, H. A., Keates, S., Gao, Y., Murikipudi, S., Mikule, K., et al. (2015). Suppression of cancer relapse and metastasis by inhibiting cancer stemness. Proc. Natl. Acad. Sci. U.S.A. 112, 1839-1844. doi: 10.1073/pnas.14241 71112

Liang, Z., Yang, N., Jiang, Y., Hou, C., Zheng, J., Shi, J., et al. (2015). Targeting docetaxel-pla nanoparticles simultaneously inhibit tumor growth and liver metastases of small cell lung cancer. Int. J. Pharm. 494, 337-345. doi: 10.1016/j. ijpharm.2015.08.042

Ling, G., Zhang, P., Zhang, W., Sun, J., Meng, X., Qin, Y., et al. (2010). Development of novel self-assembled DS-PLGA hybrid nanoparticles for improving oral bioavailability of vincristine sulfate by P-gp inhibition. J. Control. Release 148, 241-248. doi: 10.1016/j.jconrel.2010.08.010

Liu, Q., Li, R. T., Qian, H. Q., Wei, J., Xie, L., Shen, J., et al. (2013). Targeted delivery of mir-200c/doc to inhibit cancer stem cells and cancer cells by the gelatinasesstimuli nanoparticles. Biomaterials 34, 7191-7203. doi: 10.1016/j.biomaterials. 2013.06.004

Lorenzo, V. D., Giovannini, F., Herrero, M., and Neilands, J. B. (2010). Regulation of breast cancer stem cell activity by signaling through the notch 4 receptor. Cancer Res. 70, 709-718. doi: 10.1158/0008-5472.CAN-09-1681

Lu, B., Huang, X., Mo, J., and Zhao, W. (2016). Drug delivery using nanoparticles for cancer stem-like cell targeting. Front. Pharmacol. 7:84. doi: 10.3389/fphar. 2016.00084 
Luo, Y., Ziebell, M. R., and Prestwich, G. D. (2000). A hyaluronic acid-taxol antitumor bioconjugate targeted to cancer cells. Biomacromolecules 1, 208-218. doi: $10.1021 / \mathrm{bm} 000283 \mathrm{n}$

Marhaba, R., Klingbeil, P., Nuebel, T., Nazarenko, I., Buechler, M. W., and Zoeller, M. (2008). Cd44 and epcam: cancer-initiating cell markers. Curr. Mol. Med. 8, 784-804. doi: 10.2174/156652408786733667

Medema, J. P. (2013). Cancer stem cells: the challenges ahead. Nat. Cell Biol. 15, 338-344. doi: 10.1038/ncb2717

Mittal, G., and Kumar, M. N. (2009). Impact of polymeric nanoparticles on oral pharmacokinetics: a dose-dependent case study with estradiol. J. Pharm. Sci. 98, 3730-3734. doi: 10.1002/jps.21695

Mittal, G., Sahana, D. K., Bhardwaj, V., and Ravi Kumar, M. N. (2007). Estradiol loaded PLGA nanoparticles for oral administration: effect of polymer molecular weight and copolymer composition on release behavior in vitro and in vivo. J. Control. Release 119, 77-85. doi: 10.1016/j.jconrel.2007.01.016

Moffatt, S., Papasakelariou, C., Wiehle, S., and Cristiano, R. (2006). Successful in vivo tumor targeting of prostate-specific membrane antigen with a highly efficient j591/pei/dna molecular conjugate. Gene Ther. 13, 761-772. doi: 10. 1038/sj.gt.3302721

Muntimadugu, E., Kumar, R., Saladi, S., Rafeeqi, T. A., and Khan, W. (2016). Cd44 targeted chemotherapy for co-eradication of breast cancer stem cells and cancer cells using polymeric nanoparticles of salinomycin and paclitaxel. Colloids Surf. B Biointerfaces 143, 532-546. doi: 10.1016/j.colsurfb.2016.03.075

Murphy, M., and Stordal, B. (2011). Erlotinib or gefitinib for the treatment of relapsed platinum pretreated non-small cell lung cancer and ovarian cancer: a systematic review. Drug Resist. Updat. 14, 177-190. doi: 10.1016/j.drup.2011. 02.004

Nascimento, A. V., Singh, A., Bousbaa, H., Ferreira, D., Sarmento, B., and Amiji, M. M. (2015). Combinatorial-designed epidermal growth factor receptortargeted chitosan nanoparticles for encapsulation and delivery of lipid-modified platinum derivatives in wild-type and resistant non-small-cell lung cancer cells. Mol. Pharm. 12, 4466-4477. doi: 10.1021/acs.molpharmaceut.5b00642

Needham, C. J., Williams, A. K., Chew, S. A., Kasper, F. K., and Mikos, A. G. (2012). Engineering a polymeric gene delivery vector based on poly(ethylenimine) and hyaluronic acid. Biomacromolecules 13, 1429-1437. doi: 10.1021/bm30 $0145 \mathrm{q}$

Ni, M., Xiong, M., Zhang, X., Cai, G., Chen, H., Zeng, Q., et al. (2015). Poly(lactic-co-glycolic acid) nanoparticles conjugated with CD133 aptamers for targeted salinomycin delivery to $\mathrm{CD} 133^{+}$osteosarcoma cancer stem cells. Int J. Nanomedicine 10, 2537-2554. doi: 10.2147/IJN.S78498

O'Brien, C. A., Kreso, A., and Jamieson, C. H. (2010). Cancer stem cells and selfrenewal. Clin. Cancer Res. 16, 3113-3120. doi: 10.1158/1078-0432.CCR-092824

Ogris, M., Walker, G., Blessing, T., Kircheis, R., Wolschek, M., and Wagner, E. (2003). Tumor-targeted gene therapy: strategies for the preparation of ligandpolyethylene glycol-polyethylenimine/dna complexes. J. Control. Release 91, 173-181. doi: 10.1016/S0168-3659(03)00230-X

Oishi, N., and Wang, X. W. (2011). Novel therapeutic strategies for targeting liver cancer stem cells. Int. J. Biol. Sci. 7, 517-535. doi: 10.7150/ijbs.7.517

Olsson, I., Gullberg, U., Ivhed, I., and Nilsson, K. (1984). Induction of differentiation of the human histiocytic lymphoma cell line u- 937 by 1 alpha,25dihydroxycholecalciferol. Cancer Res. 43(12 Pt. 1), 5862-5867.

Pandey, S. K., Patel, D. K., Thakur, R., Mishra, D. P., Maiti, P., and Haldar, C. (2015). Anti-cancer evaluation of quercetin embedded pla nanoparticles synthesized by emulsified nanoprecipitation. Int. J. Biol. Macromol. 75, 521-529. doi: 10.1016/ j.ijbiomac.2015.02.011

Pardoll, D. M. (2012). The blockade of immune checkpoints in cancer immunotherapy. Nat. Rev. Cancer 12, 252-264. doi: 10.1038/nrc3239

Pérez-Herrero, E., and Fernández-Medarde, A. (2015). Advanced targeted therapies in cancer: drug nanocarriers, the future of chemotherapy. Eur. J. Pharm. Biopharm 93, 52-79.

Petros, R. A., and Desimone, J. M. (2010). Strategies in the design of nanoparticles for therapeutic applications. Nat. Rev. Drug Discov. 9, 615-627. doi: 10.1038/ nrd 2591

Pradhan, A., Lambert, Q. T., and Reuther, G. W. (2007). Transformation of hematopoietic cells and activation of jak2-v617f by il-27r, a component of a heterodimeric type i cytokine receptor. Proc. Natl. Acad. Sci. U.S.A. 104, 18502-18507. doi: 10.1073/pnas.0702388104
Prestwich, G. D. (2011). Hyaluronic acid-based clinical biomaterials derived for cell and molecule delivery in regenerative medicine. J. Control. Release 155, 193-199. doi: 10.1016/j.jconrel.2011.04.007

Rao, W., Wang, H., Han, J., Zhao, S., Dumbleton, J., Agarwal, P., et al. (2015). Chitosan-decorated doxorubicin-encapsulated nanoparticle targets and eliminates tumor reinitiating cancer stem-like cells. ACS Nano 9, 5725-5740. doi: $10.1021 / \mathrm{nn} 506928 \mathrm{p}$

Reya, T., Morrison, S. J., Clarke, M. F., and Weissman, I. L. (2001). Stem cells, cancer, and cancer stem cells. Nature 414, 105-111. doi: 10.1038/35102167

Riva, R., Ragelle, H., Rieux, A. D., Duhem, N., Jérôme, C., and Préat, V. (2011). Chitosan and Chitosan Derivatives in Drug Delivery and Tissue Engineering. Chitosan for Biomaterials II. Berlin: Springer.

Rosen, J. M., and Jordan, C. T. (2009). The increasing complexity of the cancer stem cell paradigm. Science 324, 1670-1673. doi: 10.1126/science.1171837

Roy, S. K., Srivastava, R. K., and Shankar, S. (2010). Inhibition of pi3k/akt and mapk/erk pathways causes activation of foxo transcription factor, leading to cell cycle arrest and apoptosis in pancreatic cancer. J. Mol. Signal. 5:10. doi: 10.1186/1750-2187-5-10

Sakae, M., Ito, T., Yoshihara, C., Iidatanaka, N., Yanagie, H., Eriguchi, M., et al. (2008). Highly efficient in vivo gene transfection by plasmid/pei complexes coated by anionic peg derivatives bearing carboxyl groups and rgd peptide. Biomed. Pharm. 62, 448-453. doi: 10.1016/j.biopha.2007.12.009

Scadden, D. T. (2006). The stem-cell niche as an entity of action. Nature 441, 1075-1079. doi: 10.1038/nature04957

Senel, S., and Mcclure, S. J. (2004). Potential applications of chitosan in veterinary medicine. Adv. Drug Deliv. Rev. 56, 1467-1480. doi: 10.1016/j.addr.2004. 02.007

Shen, S., Du, X. J., Liu, J., Sun, R., Zhu, Y. H., and Wang, J. (2015). Delivery of bortezomib with nanoparticles for basal-like triple-negative breast cancer therapy. J. Control. Release 208, 14-24. doi: 10.1016/j.jconrel.2014.12.043

Swaminathan, S. K., Roger, E., Toti, U., Lin, N., Ohlfest, J. R., and Panyam, J. (2013). Cd133-targeted paclitaxel delivery inhibits local tumor recurrence in a mouse model of breast cancer. J. Control. Release 171, 280-287. doi: 10.1016/j.jconrel. 2013.07.014

Sykes, E. A., Chen, J., Zheng, G., and Chan, W. C. (2014). Investigating the impact of nanoparticle size on active and passive tumor targeting efficiency. ACS Nano 8, 5696-5706. doi: 10.1021/nn500299p

Takebe, N., Harris, P. J., Warren, R. Q., and Ivy, S. P. (2011). Takebe n, harris pj, warren rq, ivy sptargeting cancer stem cells by inhibiting wnt, notch, and hedgehog pathways. Nat. Rev. Clin. Oncol. 8, 97-106. doi: 10.1038/nrclinonc. 2010.196

Todaro, M., D’Asaro, M., Caccamo, N., Iovino, F., Francipane, M. G., Meraviglia, S., et al. (2009). Efficient killing of human colon cancer stem cells by gammadelta t lymphocytes. J. Immunol. 182, 7287-7296. doi: 10.4049/ jimmunol.0804288

Torchilin, V. (2011). Tumor delivery of macromolecular drugs based on the epr effect. Adv. Drug Deliv. Rev. 63, 131-135. doi: 10.1016/j.addr.2010. 03.011

Vinogradov, S., and Wei, X. (2012). Cancer stem cells and drug resistance: the potential of nanomedicine. Nanomedicine 7, 597-615. doi: 10.2217/nnm.12.22

Visvader, J. E., and Lindeman, G. J. (2008). Cancer stem cells in solid tumours: accumulating evidence and unresolved questions. Nat. Rev. Cancer 8, 755-768. doi: $10.1038 / \mathrm{nrc} 2499$

Wagner, E., Zenke, M., Cotten, M., Beug, H., and Birnstiel, M. L. (1990). Transferrin-polycation conjugates as carriers for dna uptake into cells. Proc. Natl. Acad. Sci. U.S.A. 87, 3410-3414. doi: 10.1073/pnas.87.9.3410

Wang, H., Agarwal, P., Zhao, S., Xu, R. X., Yu, J., Lu, X., et al. (2015). Hyaluronic acid-decorated dual responsive nanoparticles of pluronic f127, plga, and chitosan for targeted co-delivery of doxorubicin and irinotecan to eliminate cancer stem-like cells. Biomaterials 72, 74-89. doi: 10.1016/j.biomaterials.2015. 08.048

Warburg, O., Wind, F., and Negelein, E. (1952). The metabolism of tumors in the body. Arch. Int. Pharm. Their. 88, 473-481.

Warrell, R. P. Jr., de The, H., Wang, Z.-Y., and Degos, L. (1993). Acute promyelocytic leukemia. N. Engl. J. Med. 329, 100364-100364. doi: 10.1056/ NEJM199307153290307

Wei, X., Senanayake, T. H., Warren, G., and Vinogradov, S. V. (2013). Hyaluronic acid-based nanogel-drug conjugates with enhanced anticancer 
activity designed for the targeting of cd44-positive and drug-resistant tumors. Bioconjug. Chem. 24, 658-668. doi: 10.1021/bc300632w

Williams, R. F., Sims, T. L., Tracey, L., Myers, A. L., Ng, C. Y., Poppleton, H., et al. (2010). Maturation of tumor vasculature by interferon-beta disrupts the vascular niche of glioma stem cells. Anticancer Res. 30, 3301-3308.

Wilson, T. R., Johnston, P. G., and Longley, D. B. (2009). Anti-apoptotic mechanisms of drug resistance in cancer. Curr. Cancer Drug Targets 9, 307-319. doi: 10.2174/156800909788166547

Wouters, B. G., and Koritzinsky, M. (2008). Hypoxia signalling through mtor and the unfolded protein response in cancer. Nat. Rev. Cancer 8, 851-864. doi: 10.1038/nrc2501

Wu, H., Scher, B. M., Chu, C. L., Leonard, M., Olmedo, R., Scher, G. S., et al. (1991). Reduction in lactate accumulation correlates with differentiationinduced terminal cell division of leukemia cells. Differentiation 48, 51-58. doi: 10.1111/j.1432-0436.1991.tb00242.x

Yang, N., Jiang, Y., Zhang, H., Sun, B., Hou, C., Zheng, J., et al. (2015). Active targeting docetaxel-PLA nanoparticles eradicate circulating lung cancer stemlike cells and inhibit liver metastasis. Mol. Pharm. 12, 232-239. doi: 10.1021/ mp500568z
Zahreddine, H., and Borden, K. L. (2013). Mechanisms and insights into drug resistance in cancer. Front. Pharm. 4:28. doi: 10.3389/fphar.2013.00028

Zhang, C., Gao, S., Jiang, W., Lin, S., Du, F., Li, Z., et al. (2010). Targeted minicircle dna delivery using folate-poly(ethylene glycol)-polyethylenimine as non-viral carrier. Biomaterials 31, 6075-6086. doi: 10.1016/j.biomaterials.2010.04.042

Zhou, B. B., Zhang, H., Damelin, M., Geles, K. G., Grindley, J. C., and Dirks, P. B. (2009). Tumour-initiating cells: challenges and opportunities for anticancer drug discovery. Nat. Rev. Drug Discov. 8, 806-823. doi: 10.1038/nrd2137

Conflict of Interest Statement: The authors declare that the research was conducted in the absence of any commercial or financial relationships that could be construed as a potential conflict of interest.

Copyright (c) $2017 \mathrm{Li}, \mathrm{Li}, \mathrm{Mo}$ and Dai. This is an open-access article distributed under the terms of the Creative Commons Attribution License (CC BY). The use, distribution or reproduction in other forums is permitted, provided the original author(s) or licensor are credited and that the original publication in this journal is cited, in accordance with accepted academic practice. No use, distribution or reproduction is permitted which does not comply with these terms. 